\title{
Ragang based Raga Identification system
}

\author{
Awadhesh Pratap Singh Tomer \\ Assistant Professor (Music-vocal), Department of Music Dr. H. S. G. Central University Sagar M.P. \\ Gopal Sangeet Mahavidhyalaya Mahaveer Chowk Bina Distt. Sagar (M.P.) 470113
}

\begin{abstract}
The paper describes the importance of Ragang in the Raga classification system and its utility as being unique musical patterns; in raga identification. The idea behind the paper is to reinvestigate Ragang with a prospective to use it in digital classification and identification system. Previous works in this field are based on Swara sequence and patterns, Pakad and basic structure of Raga individually.

To my best knowledge previous works doesn't deal with the Ragang Patterns for identification and thus the paper approaches Raga identification with a Ragang (musical pattern group) base model.

This work also reviews the Thaat-Raagang classification system. This describes scope in application for Automatic digital teaching of classical music by software program to analyze music (Classical vocal and instrumental). The Raag classification should be flawless and logically perfect for best ever results.

Key words: Aadhar shadaj, Ati Komal Gandhar, Bahar, Bhairav, Dhanashri, Dhaivat, Gamak, Gandhar, Gitkarri, Graam, Jati Gayan, Kafi, Kanada, Kann, Komal Rishabh, Madhyam, Malhar, Meed, Nishad, Raga, Ragang, Ragini, Rishabh, Saarang, Saptak, Shruti, Shrutiantra, Swaras, Swar Prastar, Thaat, Tivra swar, UpRag, Vikrat Swar
\end{abstract}

A Raga is a tonal frame work for composition and improvisation. It embodies a unique musical idea.(Balle and Joshi 2009, 1) Ragang is included in 10 point Raga classification of Saarang Dev, With Graam Raga, UpRaga and more. Raga created by violation of rules of GraamRaga, or/and by combination with other Ragas and /or by impurities in laws were called Ragang.(Bhagwat 2001, 33)There were 21 Ragas explained as Ragang, of which 8 were old and 13 newly created that time. Ragang were called to derive from combination in basic Ragas or changes in laws governing them. Thus many experts feel that those have no resemblance with modern Ragang. But differed view point explains that Ragas created from the shadow of Graam Raga are Ragang. 'Sinh Bhupaal' explains this in Sanskrit-

\section{"Uktanam GramaRaganam ChayaMatram Bhajantahi \\ Geetagyaee Kathita Sarvee Ragangasteen Hetuna”}

A unique phrase of notes in a Raga suggesting its characteristic feature is Ragang. Unique pattern of notes (Swaras) that are recognized easily are called Ragang named after its parent Raga. Parijaat and other texts of medieval period show the influence of this classification system. Pandit Bhavbhatt, Pandit Bhaatkande and Pandit Narayan Moreshwar khare, Krishnadhan Laxmi, S.N.Tagore elaborated and explained this method (Tomer 2012, 15).This method is useful and being followed till now because of it includes applied musical patterns. The uniqueness of group of notes in Ragang classification depends on way of using notes, tone accent, vibration, pause, slur, tie, rest, turn, appogiature and acciaccatura on different sets of notes, grace notes, difference in microtones of notes used and many more complexities. It is much more than musical pattern as we all know only words can't explain it.

Bhavbhaat was first to undertake this (seriously) and he proposed to select twenty main Thaat as primary Raga (as Ragang )(A.Popley 1921, 54). For the purpose of study Pandit Narayan Moreshwar khare explained 30 Ragang.(Tomer 2012, 15) But today from 16 to 20 are in use. Kafi Thaat is having the most numbers of popular Ragangs like Kafi, Dhanashri, Sarang, Malhar and Kanada. (Devangan 2010, 291) To understand the applicability, speciality and deepness of Ragang, the study of Kafi Thaat -Ragang is very important and useful. Both the forms of Gandhar and Nishad (sharp and flat) are used in many ways in Kafi, Dhanashri, Saarang, Malhar, Bahar and Kanada .

Difference of Swar forms to the extent of microtones (shrutiantra) is always a point of discussion in many Ragas. Same Swar are used in different Raga differently like Ati Komal Gandhar and Dhavat in Raga Darbari Kanara, Rishabh in Shree Raga, double Nishad in Malhar, Rishabh in Raga Sankara, double Madhyam in Lalit. These minute differences in use of notes (Shrutiantra) can be easily expressed and explained by Ragang. Some patterns of notes are very special/unique in Raga and Ragang and are known as the identity of the same like re pa with Meed is alive part of Malhar, pa re is alive part of Kalyan, Likewise vibration in Komal Rishabh and Komal Dhaivat and Madhyam to Komal Rishabh with Gitkarri (Ma (Ma) Rr\} in Bhairav 
Raga, "Ga Ma Dha Sa" with less use of Pancham and Nishad in ascending order sliding to Sadaj with vibration of Dhaivat is the main set of notes in ascending order (Paranjpe 1986, 67).

The Ragang system should be combined actively in Thaat system. This Thaat-Ragang concept should be elaborated and extended to overcome the limitation of both the system. Without increasing the Thaat in large numbers, the system can be made more logical and micro pure by including Ragang in it. Ragang should be studied minutely to search the opportunity of better and flawless classification system. Special set of notes or special pattern of Swaras is a key to the Thaat-Ragang classification system.

The specific structure and format of Ragang system increases the possibilities of different uses of it. The sets of Ragang Ang are used many times in the Raga of the same Ang during systematic rendering of notes. Relation of different notes with Ragang Ang is predefined. Thus all the notes are arranged in definite order, proportion and pattern. These variable patterns in specific Raga are recognized with the help of Ragang. The set of notes of Ragang Ang and there proportion with other notes can be defined by graph patterns or alternatives method of sound analysis in digital method. Recordings of specific Ang and pattern can be compared and turned into digital patterns by HMM, PCDs, PCDDs, SVMs and other methods used by previous researchers. Even live singing can be compared automatically by machines/computers to define its Ragang and Raga.

\section{PREVIOUS WORKS IN RAGA IDENTIFICATION \& CLASSIFICATION}

One of the earlier works on Raga classification is by Sahasrabuddhe (Sahasrabuddhe and R.Upadhye 1992) who modeled a Raga as a finite state automaton based on the Swara patterns followed in it. Pandey (Pandey, Mishra and Ipe 2003)extended this idea of Swara sequence working with Hidden Markov Models on Swara sequences extracted using a heuristics driven note-segmentation technique. They employed a novel Pakad matching algorithm that improved the HMM based results. Much work published and presented in this field is on Raga identification or detection is by Pitch-Class Distribution (PCDs) and Pitch-class Dvad Distribution (PCDDs) which represent the probabilities of the dyads. Chrodia and Rae derived PCD and PCDD from Harmonic Pitch Class Profiles (HPCP) and used these distributions for classification using SVMs. They achieved results with accuracies of $78 \%$ for PCDs and $97.1 \%$ for PCDDs. Sridhar and Geetha (Sridhar and Geetha 2009) used template matching for the task of raga identification.

Another new approach is performing Raga identification without the knowledge of the scale of the performance is by using Swara based features extracted from the chromagram using the concept of Vadi (Most prominent Swara of Raga) and perform discrete HMM based and Classification Tree based experiments on the data.

Experts and analysts have achieved results with high accuracy using the above approaches. But my approache differs on the matter of use of Thaat-Ragang classification system for Raga Classification and Recognition system.

\section{METHOD OF RAGA CLASSIFICATION BY THAAT-RAGANG}

Fine arts and performing arts are also becoming the parts of digital world but are not created, edited and managed fully by Digital programming and virtual logics. Humans have the intelligence and creativity which computers lack.

Music is the finest of all fine arts as it involves knowledge, reasoning, practice, talent, creativity, extempore, courage to face audience. On stage creativity and spontaneous creation with stable temperament which is not needed in fine arts and theater as they are designed and created much before the performance and display. This is the main reason because of which music is not yet created, classified, corrected and logically examined by computer processors. Indian music is only edited with many limitations and mixed in studios and stored by music industry and individuals. Digital instruments and software are now also helping us to create music but the creativity is all ours. After so much research, no such classification or recognition software which is professionally acclaimed is commercially available.

Ragang and Musical Patterns defining the basic rule of raga elaboration and successive renderence or presentation can be digitalized in music software. Digital storage and maintenance is important for music and its future. Raga music in Indian classical music is governed by rules of Thaat and Ragang. These classification rules can also be used in digital classification through recognition of notes and Ragang patterns. So the Ragang Patterns and sets of notes will help in comparing and evaluating the presentation of students digitally. The clips examined by software is feeded and the predefined sets of notes and musical patterns based on Ragang and Thaat rules will help the software to evaluate the clip logically resulting to either correction in the wrong part of clip or showing error for the same. The proposed software or digital program will act as a digital guru and even a step ahead to correct the clip by itself. Similar softwares are under development and in the process of evaluation. Those programs lack accuracy because they are not designed keeping Ragang patterns in centre. The rules and standard in Ragang pattern should be feed exactly the same as originally rendered by experts and Gurus. For example the advanced software in chess includes all the possible tricks and even more then that that 
a human can think buts such a software for Indian ragas is not available which can produce all the possible Swarvistar of a raga possible to be played and performed.

This software can help new students to correct themselves with the help of the software. Raga can be easily classified digitally for making playlists, curriculum support study material, time based listening etc.

\section{IMPORTANT POINTS OF STUDY IN SOFTWARE DEVELOPMENT}

- Indian natural scale (Saptak) and western equally tempered scale is different. Vikrat Swar differs in many Ragas as variants of Tivra and Komal Swar as those are used in different Ragang. Promoting these Aati komal or Tivra swar with the help of software and digital program will help national and international music fraternity to know and feel the original effects of ragas. The exact Swar and Shruti with Meed, Gamak, Kann and other effects should be included in software according to different Raga and Ragang.

- It is noticed that the developers of these music software's have less knowledge of music and even they do not consult enough experts. This software should incorporate views and vocals inputs of as many experts as possible.

- This analytical software should strictly follow Indian Swars, Raga and special musical patterns same as they are generally used and noted in notation system. Efforts can be made so that the Swars are displayed in Devenagri (Hindi Script) so that it will be accepted and acclaimed by Indian music professionals worldwide.

- Using this software students may be taught and corrected, even demonstration can be made by this program. Logical inputs should be perfect in it so that the performance of this software is according to the structure of Raga and Swar Prastar.

- 30 Ragang and 10 Thaat inclusively will form a web of musical patterns in which Ragas will flourish and remain neat and pure. The Raga which will not match the set of Ragang or Thaat will automatically grouped in new Ragang or Thaat of the name of its own.

- Including the views of different experts of different school will automatically make a consensus on issues of conflicts and different at various points.

- Vocal and Instrumental inputs will also make a suggestive playlist for students and guide to know the desired Raga.

Direct applications of Swar and Shruti (tones and micro tones) can be seen in Indian music only with the help of Ragang elaboration. Ragang will become more practical and logical with the help of Thaat Ragang concept of classification (software based on). Importance of Ragang will increase in theory books of music and in Raga description in books and demonstration. Original and perfect structure of Raga will appear before students, teachers and experts as well.

New generation is learning classical music with the help of Harmonium or synthesizers and they do not know the original Swars; that's why it is very difficult to acquire, adapt, and perform Indian music even after adequate inborn talent and extensive efforts. Many times Harmonium and other such instruments are not properly turned and students practice Ragas with accompaniments of such instruments. When the basic Swar are not true, how can the Ragas learned will be perfect? These students sing the Harmonium Swar for whole of their life and some of them even become music teacher and carry forward the un-tuned tradition (Asur Parampara) to others. This proposed software will help to correct them.

Thaat-Ragang Digital Classification and Recognition system will help to retain and regain the original "Bharatiya Raga Sangeet", which is world famous for its mystic effects from Tansen and Beju till now.

\section{Bibliography:-}

[1]. A.Popley, Herbert. The Music of India. London: Oxford university Press, 1921.

[2]. Balle, Shreayas, and Rushikesh Joshi. "Raga identification by using swara intonation." Journal of ITC SRA-1, $2009: 1$.

[3]. Bhagwat, Ashwani. "Sangeet Ratnakar me pratyach va apratyach Raga Vargikaran." Edited by L.N.Garg. Sangeet Sahastrabdi ank, 2001: 31 .

[4]. Chudhari, Subhash Rani. Sangeet ke pramukh sastriya sidhhant. Delhi: Kanishka Publishers, 2008.

[5]. Devangan, Tulsi ram. Bhartiya sangeet shastra. Bhopal: MP Hindi Geanth Acadamy, 2010.

[6]. Pandey, G., C. Mishra, and P. Ipe. "Tansen: A system for automatic raga identification." Proc. of Indian International Conference on Artificial Intelligence. 2003. 1350-1363.

[7]. Paranjpe, Sharatchandra. Sangeet Bodh. bhopal: Madhya Pradesh Hindi Granth Academy, 1986.

[8]. Sahasrabuddhe, H., and R.Upadhye. "On the computational model of raag music of indian." work shop on $\mathrm{Al}$ and music. European confrence, 1992.

[9]. Sridhar, R., and T. Geetha. "Raga identification of carnatic music for music information retrieval." International Journal of recent trends in Engineering Vol. 1, No.1, 2009: 571-574.

[10]. Tomer, Awadhesh pratap singh. Sangeet Shastra Sarita. sagar: Krishna computer, 2012. 